\title{
Intratumoral injection of dendritic cells overexpressing interleukin-12 inhibits melanoma growth
}

\author{
WEIRONG YAO $^{1 *},{\text { YINGLIANG } \text { LI }^{2 *}, \text { LIN ZENG }^{1}, \text { XIQUAN ZHANG }}^{1}$, \\ ZHIYONG ZHOU ${ }^{1}$, MEIXUE ZHENG ${ }^{3}$ and HUIPING WAN ${ }^{1}$ \\ ${ }^{1}$ Oncology Department, Jiangxi Provincial People's Hospital; \\ ${ }^{2}$ Department of General Surgery, The First Affiliated Hospital of Nanchang University; \\ ${ }^{3}$ Graduate School, Nanchang University, Nanchang, Jiangxi 330006, P.R. China
}

Received January 25, 2019; Accepted April 18, 2019

DOI: 10.3892/or.2019.7165

\begin{abstract}
The present study aimed to investigate the antitumor effects of an intratumoral injection of dendritic cells (DCs) overexpressing interleukin-12 (IL-12) on melanoma. DCs, isolated from mouse spleen, were gene-modified using an IL-12 overexpression vector. Melanoma B6 cells were injected into C57BL/6 mice to generate tumors. Thereafter, DCs overexpressing IL-12 were injected into the tumors, and tumor volume was subsequently measured. Pathological changes in tumor tissue were detected by hematoxylin and eosin staining. The expression of interleukin-4 (IL-4) and IL-12 in tumors was measured by enzyme-linked immunosorbent assay, real-time PCR and western blotting. DCs were successfully isolated and a lentivirus vector expressing IL-12 was constructed. After intratumoral injection of phosphate-buffered saline (control group), tumor cells exhibited malignant growth; whereas tumors injected with DCs (DC group) or DCs + empty vector (DC + vector group) exhibited a small amount of inflammatory cell infiltration and limited areas of tissue necrosis. In contrast, tumors injected with DCs overexpressing IL-12 (DC + IL-12 group) displayed severe tissue necrosis, loss of cell structure, and inflammatory cell infiltration. Compared with the control group, the tumor volumes were significantly lower in the DC, the DC + vector and the DC + IL-12 groups, while the expression of IL-12 and IL-4 in the tumors was significantly higher. Importantly, the most marked changes in tumor volume and IL-12 and IL-4 expression were in the DC + IL-12 group, which were significantly greater than those in tumors treated with unmodified DCs. Hence, intratumoral injection of DCs overexpressing IL-12 exerted strong antitumor effects in
\end{abstract}

Correspondence to: Dr Huiping Wan, Oncology Department, Jiangxi Provincial People's Hospital, 92 Aiguo Street, Nanchang, Jiangxi 330006, P.R. China

E-mail:wan1huiping@163.com

*Contributed equally

Key words: melanoma, dendritic cells, interleukin-12, interleukin-4 melanoma, and biotherapy with DCs overexpressing IL-12 is a potential treatment strategy for melanoma.

\section{Introduction}

Melanoma is a malignant tumor that originates from cells that produce melanin. It is characterized by the sudden appearance, or rapid growth, of moles with deepening color and comet-like structure. The symptoms of this type of cancer include local pain, infection, ulcer or bleeding and enlarged lymph nodes (1). Melanoma tumors occur most often in the lower limbs, followed by the head, neck, upper limbs and eyes. Early metastasis occurs via lymphatic and blood vessels to the liver, brain, bone and mucosa, among other tissues (2). Biotherapy is a new choice for tumor treatment (3) and is characterized by fewer complications and less toxic and side effects than traditional therapeutic approaches (2). Biotherapy can be used alone or as complementary treatment following surgery, radiotherapy and chemotherapy (4). Hence, biotherapy is considered an important development in the treatment of malignant tumors, particularly melanoma (5).

Dendritic cells (DCs), the most powerful antigen presenting cells (APCs) in vivo and the only APCs that can activate $\mathrm{T}$ lymphocytes, have an important role in connecting innate and adaptive immunity (6). Their function is to present pathogen-derived antigen peptides, via major histocompatibility molecules (MHC), to immature $\mathrm{T}$ lymphocytes in lymphoid organs. Through this process, DCs form the key link between innate and adaptive immunity, which is essential for antigen-specific immune responses (7).

IL-12 is an immunoregulatory cytokine that functions as an important link in the process of immune regulation (8). IL-12 can inhibit tumor growth through the following pathways: Stimulation of $\mathrm{T}$ cell activation and promotion of the transformation of naïve $\mathrm{T}$ cells into $\mathrm{T}$ helper type 1 cells; activation of cytotoxic $\mathrm{T}$ lymphocytes (CTLs) and natural killer cells and promotion of the secretion of tumor necrosis factor (TNF)- $\alpha$ and other cytokines (9). Moreover, IL-12 can upregulate the levels of vascular adhesion molecule-1 and cadherin, induce apoptosis and promote neovascularization (10-12). Hence, IL-12 is a promising immunotherapeutic agent that links innate and adaptive immunity (13). IL-12 has 
been observed to have antitumor effects in several preclinical and clinical studies (14-16). In the present study, monocytes were isolated and differentiated into mature DCs, which were then transfected with lentiviruses constructed to overexpress IL-12. Thereafter, these genetically modified DCs were directly injected into melanoma tumors in model mice, and their antitumor effects were evaluated.

\section{Materials and methods}

Preparation of DCs. Twenty specific pathogen-free male C57BL/6 mice (weighing $22 \mathrm{~g} ; 4$ weeks old) were purchased from Changzhou Cavans Laboratory Animal Co. Ltd. [license no. SCXK (Su) 2016-0010] and housed in a specific pathogen-free condition that was automatically maintained at a temperature of $23 \pm 2^{\circ} \mathrm{C}$, a relative humidity of $45-65 \%$, and with a controlled $12 \mathrm{~h}$ light/dark cycle and access to food and water ad libitum. Mice were sacrificed by cervical dislocation following anaesthesia with isoflurane, immersed in $75 \%$ ethanol for 5-10 min, and then their spleens were removed by aseptic surgery, and the tissues were soaked in Hank's solution (Gibco; Thermo Fisher Scientific, Inc., Waltham, MA, USA). Spleens were rinsed repeatedly until they turned white. Then, cell suspensions were collected, and mononuclear cells were obtained by density gradient centrifugation (987 x g, for $20 \mathrm{~min}$ ), using lymphocyte separation solution (C-44010; Bio-Connect B.V., Huissen, The Netherlands), and then washed twice with phosphate-buffered saline (PBS) by centrifugation. Spleen mononuclear cell density was adjusted to $1 \times 10^{7} / \mathrm{ml}$, using lymphocyte separation solution, and cells were plated into 6 -well tissue culture plates $(2 \mathrm{ml} /$ well $)$, and incubated at $37^{\circ} \mathrm{C}$ in a $5 \% \mathrm{CO}_{2}$ incubator. After $2 \mathrm{~h}$, medium was aspirated from the suspended cells, and replaced with Dulbecco's modified Eagle's medium, containing 10\% fetal bovine serum (FBS), and supplemented with granulocyte macrophage colony-stimulating factor (GM-CSF) and IL-4 $(0.1 \mathrm{~g} / 1)$. On the 7 th day, lipopolysaccharides (LPS) were added to the medium at a final concentration of $1 \mu \mathrm{g} / 1$, and after $24 \mathrm{~h}$ of further incubation, cells were collected. Antibodies against CD40 (cat. no. 553723), CD80 (cat. no. 561955) and CD86 (cat. no. 561962) (all from BD Biosciences, Franklin Lakes, NJ, USA) were used to identify mature DCs by flow cytometer method as previously described (6). The experimental protocols were approved by the Ethics Committee of Jiangxi Provincial People's Hospital (Nanchang, China).

Construction of the IL-12 overexpression vector. The $I L-12$ gene sequence was identified in the NCBI database (https://www. ncbi.nlm.nih.gov/gene/16159) and cloned into the EcoRI and $B s t$ BI restriction enzyme sites of the pCDH-CMV-MCS-EF1CopGFP-T2A-Puro lentivirus vector (CD513B-1-SBI; System Biosciences LLC, Palo Alto, CA, USA).

Tumor model and experimental groups. Mouse melanoma cells were purchased from the Cell Bank of the Shanghai Academy of Sciences (Shanghai, China) and cultured in RPMI-1640 medium (HyClone; GE Healthcare, Chicago, IL, USA). Cultures were supplemented with $10 \%$ fetal bovine serum (FBS) (SKU: 04-007-1A; Biological Industries, Kibbutz Beit-Haemek, Israel) and $100 \mathrm{U} / \mathrm{ml}$ penicillin-streptomycin (P1400; Beijing Solarbio
Science \& Technology Co., Ltd., Beijing, China), in 5\% $\mathrm{CO}_{2}$ at $37^{\circ} \mathrm{C}$. Cells used in the experiments exhibited $\sim 70 \%$ confluence. B16 cells $\left(1 \times 10^{7}\right)$ were subcutaneously administered into the right forelimb armpit of each mouse $(\mathrm{C} 57 \mathrm{BL} / 6$, male, 4-weeks old). Twelve days after injection, tumors were formed, and mice were randomly divided into four groups: i) Tumors injected with PBS (controls); ii) tumors injected with untreated DCs (DC group); iii) tumors injected with DCs transfected with empty vector (DC + vector group); and iv) tumors injected with DCs transfected with vector overexpressing IL-12 (DC + IL-12 group). Different types of DCs (untreated, transfected with lentiviral vector alone, and transfected with DCs overexpressing IL-12) were injected into the tumors on the first, third, and seventh days after tumor formation; each mouse received $1 \times 10^{6}$ DCs diluted in $0.2 \mathrm{ml}$ PBS. One week after treatment, mice were sacrificed by cervical dislocation, fixed on a sterile towel sheet, and the hair was removed around the tumor to fully expose it. Tumors were then collected and their volumes measured according to formula: $\mathrm{V}=\left(\right.$ length $\mathrm{x}$ width $\left.{ }^{2}\right) / 2$ as previously described (17). According to IACUC guidelines, all the tumour volumes did not exceed $4.2 \mathrm{~cm}^{3}$.

Hematoxylin and eosin $(H \& E)$ staining. Tumors were fixed in $4 \%$ paraformaldehyde overnight at $4^{\circ} \mathrm{C}$. For staining, tissues were washed with water for several hours, and then dehydrated in 70, 80 and $90 \%$ ethanol, xylene, and other mixture for $15 \mathrm{~min}$, and then in xylene I for $15 \mathrm{~min}$, II for 15 min, until transparent. Samples were then placed in a mixture of xylene and paraffin (1:1) for $15 \mathrm{~min}$, and then paraffin was added for 50-60 min. The tissues were then paraffin-embedded and sections were mounted on slides, dewaxed and rehydrated. Then, the sections were stained with hematoxylin for $3 \mathrm{~min}$ and eosin for $3 \mathrm{~min}$ at room temperature and observed by an Olympus BX51 light microscopy (Olympus Corp., Tokyo, Japan).

Enzyme-linked immunosorbent assay (ELISA). IL-12 and IL-4 in tumor tissues were detected using ELISA kits (m1037868 and $\mathrm{m} 1002149$, respectively; mlbio, Shanghai, China), following the manufacturer's instructions.

Quantitative polymerase chain reaction ( $q P C R$ ). Total RNA was extracted from tumors using an UltraPure RNA Extract kit (CW0581M; CWBio, Shanghai, China). cDNA was synthesized and used as a template for the detection of mRNA expression. GAPDH was used as an internal reference to calculate $I L-12$ and $I L-4$ levels in each group. Reactions included $9.5 \mu \mathrm{l}$ RNase-Free $\mathrm{dH}_{2} \mathrm{O}, 1 \mu \mathrm{l}$ cDNA/DNA, $2 \mu \mathrm{l}$ primers, and 12.5 $\mu 1$ 2X GoldStar Taq Master Mix (CW0960; CWBio) and were conducted using the following temperature cycles: $95^{\circ} \mathrm{C}$ (denaturation) for $10 \mathrm{sec}, 53^{\circ} \mathrm{C}$ (annealing) for $30 \mathrm{sec}$, and $72^{\circ} \mathrm{C}$ (extension) for $60 \mathrm{sec}$, for 40 cycles. The primers were: $I L-12$ forward, (5'-TCCAGAGCCACCTCA AAAC-3') and $I L-12$ reverse, (5'-CGTATGCGGAAGTGA AGAAG-3'); $I L-4$ forward, (5'-TGTCATCCTGCTCTTTTT CTC-3') and $I L-4$ reverse, (5'-GTGGTGTTCTTCGTTGCT GT-3'); GAPDH forward, (5'-AAGAAGGTGGTGAAGCAG G-3') and GAPDH reverse, (5'-GAAGGTGGAAGAGTGGGA GT-3'). The $2^{-\Delta \Delta C q}$ method was used to quantify the results as previously described (18). 

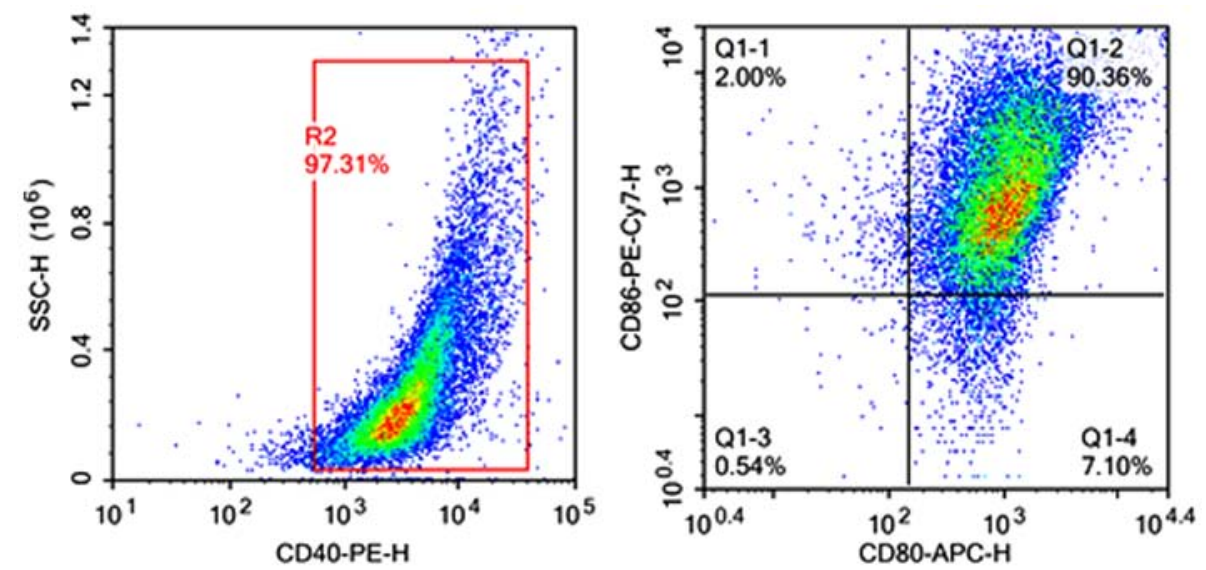

Figure 1. Confirmation of DC isolation by flow cytometry, based on the expression of CD40, CD80 and CD86. The positive expression rate of CD40 in DCs was $97.31 \%$, and those for CD80 and CD86 were $>90 \%$. DCs, dendritic cells.

Western blotting. Proteins were isolated from tumor tissues using a protein isolation kit (C1053; Applygen Technologies Inc., Beijing, China) $\left(4^{\circ} \mathrm{C}\right.$ for $30 \mathrm{~min}, 8,766 \mathrm{x}$ g for $\left.10 \mathrm{~min}\right)$. Protein concentrations were determined using a BCA kit (Thermo Fisher Scientific, Inc.). Proteins were subjected to $12 \%$ sodium dodecyl sulfate polyacrylamide gel electrophoresis and transferred onto nitrocellulose membranes. Membranes were then blocked using 5\% skimmed milk, and incubated with the following primary antibodies overnight at $4^{\circ} \mathrm{C}$ : Mouse monoclonal anti-GAPDH (dilution 1:2,000; cat. no. TA-08; ZSBIO, Beijing, China); rabbit polyclonal anti-IL-4 (dilution 1:1,000; bs-2018R, BA0980); or rabbit polyclonal anti-IL-12 (dilution 1:1,000; cat. no. ab106270; Abcam). Then membranes were incubated with secondary antibody (HRP-labeled goat anti-rabbit IgG; dilution 1:200; cat. no. A16104SAMPLE; Thermo Fisher Scientific, Inc.) for 1-2 h at room temperature. Subsequently, ECL exposure solution was used to identify specifically bound proteins and images were generated by exposure of the membranes to X-ray film. Grayscale values were analyzed using Quantity One software (Bio-Rad Laboratories, Inc., Hercules, CA, USA).

Statistical analysis. All data are presented as means \pm standard deviation (SD), and were statistically analyzed using the SPSS 19.0 (IBM Corp., Armonk NY, USA), using one-way analysis of variance (ANOVA) followed by Bonferroni testing. $\mathrm{P}<0.05$ was considered to indicate a statistically significant difference.

\section{Results}

Confirmation of DC isolation. To confirm that we successfully isolated DCs from mouse spleen, we conducted flow cytometry. Isolated DCs had CD40-positive expression rates of $97.31 \%$, and were $>90 \%$ positive for CD80 and CD86 (Fig. 1). These results indicate that DCs were successfully isolated.

Verification of IL-12 overexpression. As shown in Fig. 2, IL-12 expression was significantly increased in DCs transfected with the IL-12 overexpression vector compared with the controls $(\mathrm{P}<0.05)$, while transfection with the vector alone did not alter IL-12 expression levels.

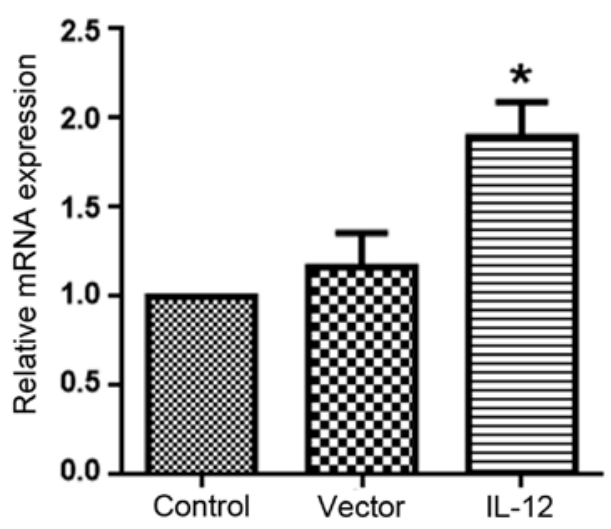

Figure 2. Verification of IL-12 overexpression. IL-12 expression was significantly increased in the DCs transfected with the lentivirus overexpressing IL-12, compared with the control group, while transfection of the vector alone did not affect IL-12 expression. Results are expressed as means \pm SD. ${ }^{*} \mathrm{P}<0.05$ compared with controls (one-way ANOVA). IL-12, interleukin-12; DCs, dendritic cells.

Tumor volumes in each group. To evaluate the effects of IL-12 on tumor growth, tumor volumes were measured. The tumor volumes in each group are presented in Fig. 3. Compared with the control group, tumor volumes in the DC, the DC + vector, and the DC + IL-12 groups were significantly decreased $(\mathrm{P}<0.05$ vs. control). Furthermore, the tumor volume was most markedly decreased in the DC + IL-12 group ( $\mathrm{P}<0.05$ vs. DC).

Morphological changes. Next, we examined the morphology of tumors in the experimental groups, to determine the effects of DCs overexpressing IL-12. As shown in Fig. 4, there was clear proliferation of tumor cells in the control group. Small amounts of inflammatory cell infiltration and limited areas of tissue necrosis were observed in the DC and DC + vector groups; however, in the DC + IL-12 group, tumors exhibited a loss of cell structure in necrotic foci and inflammatory cell infiltration at the edge of necrotic foci, with a large number of inflammatory cells and a few adipocytes infiltrating the margins of the necrotic regions.

IL-12 and IL-4 expression. To evaluate the expression levels of IL-12 and IL-4 in each experimental group, we conducted 

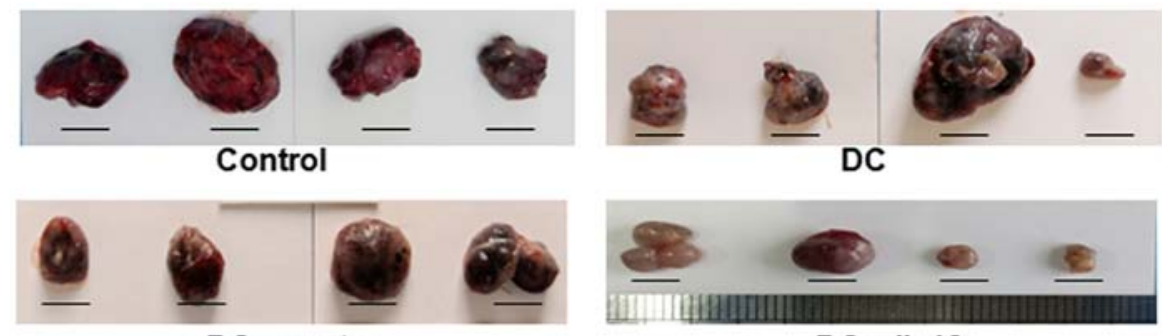

$\mathrm{DC}+$ vector

$\mathrm{DC}+\mathrm{IL}-12$

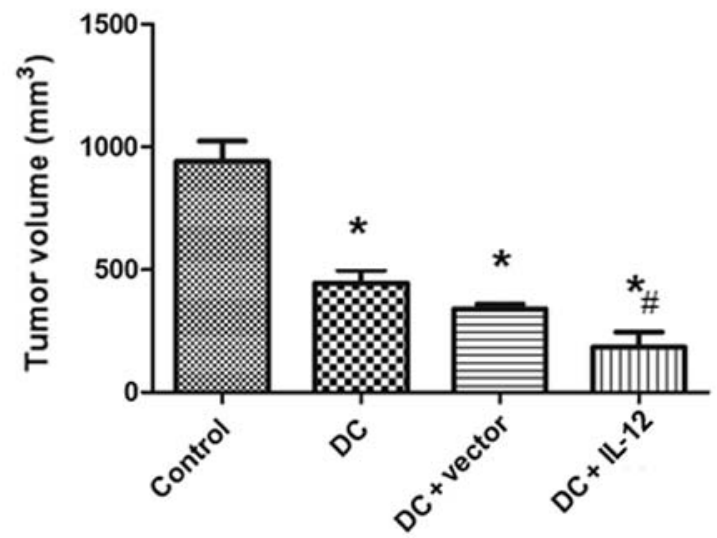

Figure 3. Tumor volumes in each group. Compared with the control group, tumor volumes were significantly lower in the DC, the DC + vector and DC + IL-12 groups, and the volume of tumors in the DC + IL-12 group were most markedly decreased. Results are presented as means \pm SD. "P $<0.05$ compared with the control; ${ }^{\#} \mathrm{P}<0.05$ compared with the DC group (one-way ANOVA). Scale bar, $5 \mathrm{~mm}$. DC, dendritic cell; IL-12, interleukin-12.

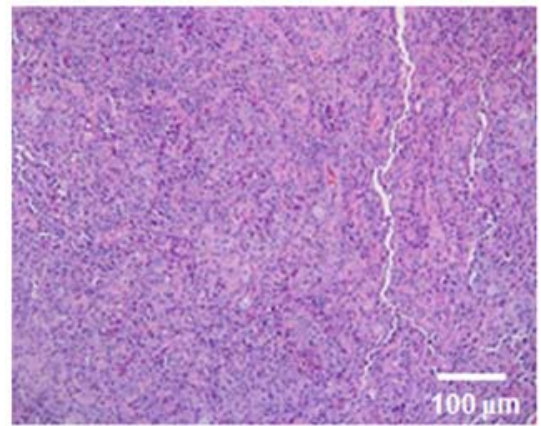

Control

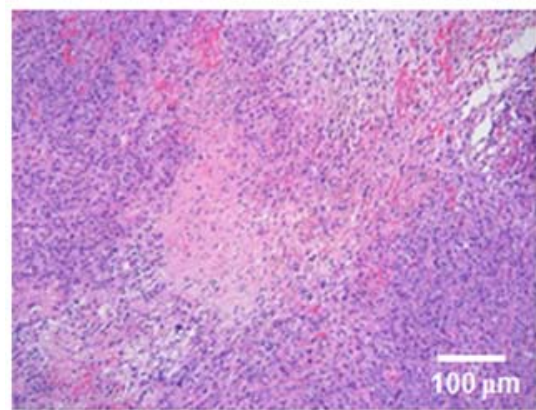

$\mathrm{DC}+$ vector

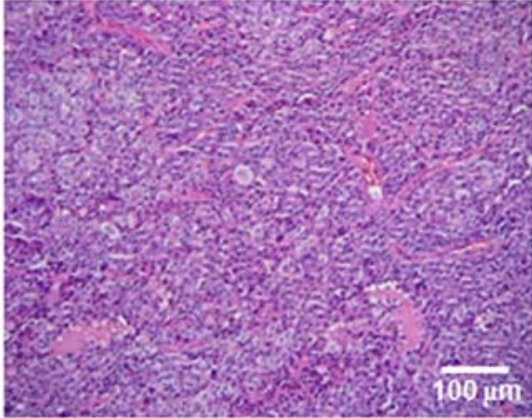

DC

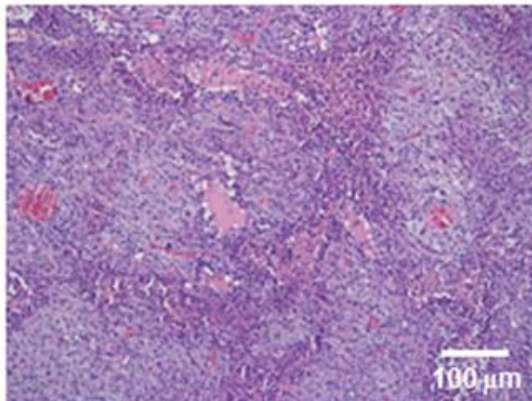

$\mathrm{DC}+\mathrm{IL}-12$

Figure 4. Morphological changes. Abnormal proliferation of tumor cells was observed in the control group. A small amount of inflammatory cell infiltration and limited areas of tissue necrosis were observed in the DC and DC + vector groups. In the DC + IL-12 group, the tumors exhibited a loss of cell structure in the necrotic foci, with infiltration of a large number of inflammatory cells, along with a few adipocytes, at the margins of the necrotic foci. DC, dendritic cell; IL-12, interleukin-12.

ELISAs, qPCR and western blotting. Compared with the control group, expression of IL-12 and IL-4 was significantly higher in the DC, the DC + vector and the DC + IL-12 groups, as evidenced by ELISA ( $\mathrm{P}<0.05$ vs. control) (Fig. 5), real-time PCR ( $<<0.05$ vs. control) (Fig. 6) and western blotting $(\mathrm{P}<0.05$ vs. control) (Fig. 7). Expression levels of IL-12 and 

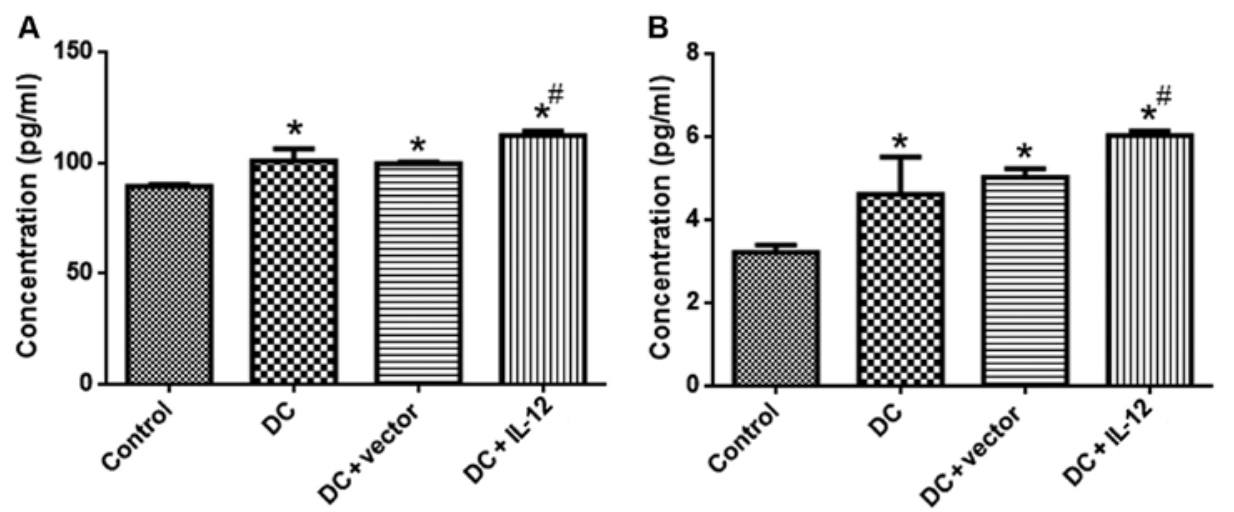

Figure 5. IL-12 and IL-4 expression in tumor samples, as detected by ELISA. (A) IL-12; (B) IL-4. Compared with the control group, expression levels of IL-12 and IL-4 were significantly increased in the DC, the DC + vector and the DC + IL-12 groups. The expression of IL-12 and IL-4 was most markedly increased in the DC + IL-12 group. Results are presented as means $\pm \mathrm{SD}$. ${ }^{*} \mathrm{P}<0.05$ compared with control group; ${ }^{*} \mathrm{P}<0.05$ compared with the DC group (one-way ANOVA). DC, dendritic cell; IL-12, interleukin-12; IL-4, interleukin-4.
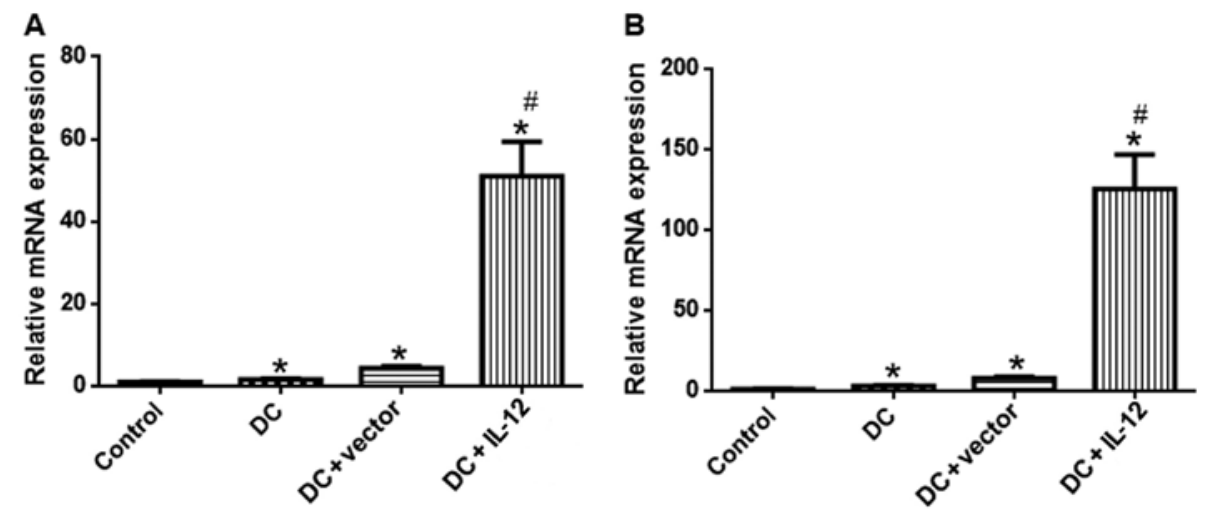

Figure 6. $I L-12$ and $I L-4$ expression detected by qPCR. (A) $I L-12$; (B) $I L-4$. Compared with control group, expression levels of $I L-12$ and $I L-4$ were significantly higher in the DC, the DC + vector the and DC + IL-12 groups. The expression of $I L-12$ and $I L-4$ was most markedly increased in the DC + IL-12 group. Results are presented as means $\pm \mathrm{SD}$. ${ }^{*} \mathrm{P}<0.05$ compared with the control group; ${ }^{*} \mathrm{P}<0.05$ compared with the DC group (one-way ANOVA). DC, dendritic cell; IL-12, interleukin-12; IL-4, interleukin-4.
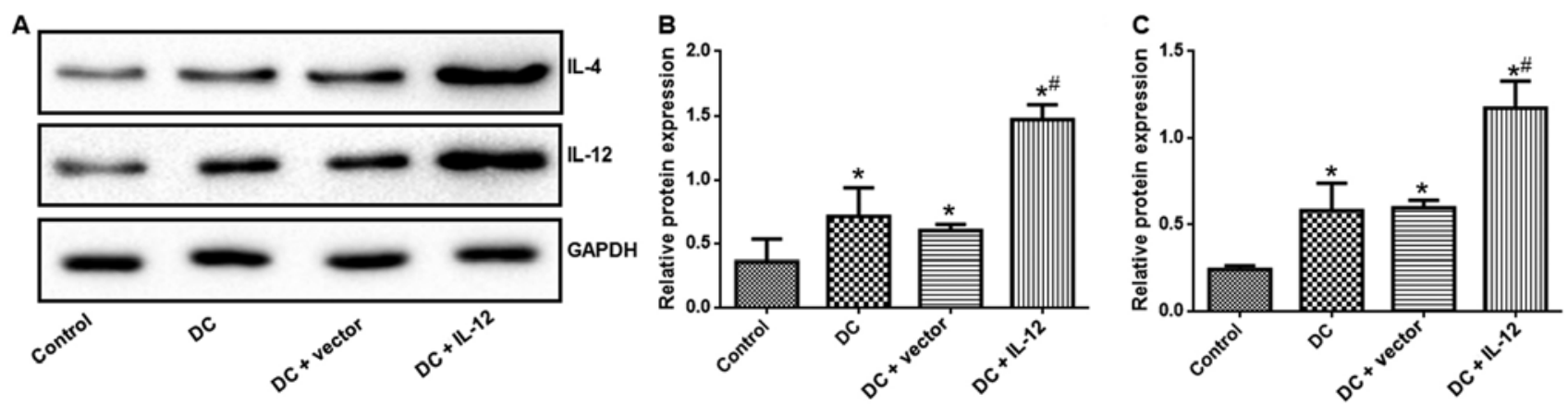

Figure 7. IL-12 and IL-4 expression detected by western blotting. (A) Representative blots, (B) IL-12 and (C) IL-4. Compared with control group, levels of IL-12 and IL-4 were significantly increased in the DC, the DC + vector, and the DC + IL-12 groups. Levels of IL-12 and IL-4 were most markedly increased in the DC + IL-12 group. Results are presented as means $\pm \mathrm{SD}$. ${ }^{*} \mathrm{P}<0.05$, compared with the control group; ${ }^{~} \mathrm{P}<0.05$ compared with the DC group (one-way ANOVA). DC, dendritic cell; IL-12, interleukin-12; IL-4, interleukin-4.

IL-4 were most markedly increased in the DC + IL-12 group ( $\mathrm{P}<0.05$ vs. DC).

\section{Discussion}

In the present study, monocytes were isolated and induced to develop into mature dendritic cells (DCs), which were then transfected with a vector overexpressing interleukin-12 (IL-12). Intratumoral injection of gene-modified DCs inhibited the growth of melanoma tumors. The possible mechanisms involved relate to the immunotherapeutic effects of interleukin-4 (IL-4). This study is the first to support the antitumor effects of DCs overexpressing IL-12 in melanoma tumors. 
Melanomas are skin tumors related to trauma, and neovascularization and endothelial growth factor have key roles in their development. Structural and functional abnormalities are markers of neovascularization in this type of tumor, and identification of effective treatments that can cure this disease is the subject of intense research (19). With the continuous development of immunological methods, biological immunotherapy has achieved impressive results in clinical practice. Simultaneously, gene therapy has become a promising method for cancer treatment. DCs are the most powerful APCs and can initiate the majority of immune responses (20). Due to lack of understanding of the origin and differentiation of DCs, the number of cells that can be obtained is very small, which greatly limits study of their functional characteristics. Therefore, it is crucial to establish technology to enable the culture of mature DCs in vitro, to facilitate the generation of sufficient numbers of functional DCs.

In this study, granulocyte-macrophage colony-stimulating factor $(G M-C S F)$, and IL-4 were used to induce DC differentiation in vitro. GM-CSF is required for maintenance of the differentiation, development, survival and function of DCs (21). GM-CSF mainly promotes the development of myeloid cells and differentiation of hematopoietic stem cells (HSCs) into DCs, monocytes and macrophages, with primarily monocytes produced (22). IL-4 can increase and stabilize the expression of the CD molecules induced by GM-CSF. Treatment with IL-4 and a very low concentration of GM-CSF can stimulate DCs, promote their maturation, and upregulate the expression of MHC-II and costimulatory molecules. IL-4 can also promote the secretion of IL-12, and maintain DCs in an immature state (23). In this study, we induced the generation of mature DCs using this method, which were then identified by flow cytometry.

IL-12 is a newly discovered cytokine with strong antitumor effects that can be produced by B cells, mast cells, neutrophils, and thymic stromal cells, but is primarily secreted by DCs (24). IL-12-transfected DCs have exhibited clear antitumor effects on melanoma, kidney and glioma (25-27). Furthermore, IL-12 can induce mouse erythroleukemia cells to differentiate into DCs, and the differentiated cells were found to have antigen presenting function (28). The results of this study showed that DCs overexpressing IL-12 can reduce melanoma tumor volumes. Furthermore, our pathological findings demonstrated that tumors treated with DCs overexpressing IL-12 had enlarged necrotic surfaces, with large numbers of inflammatory cells at the margins of necrotic foci. These results suggest that IL-12-transfected DCs can inhibit the growth of tumors and have clear antitumor effects.

IL-4 promotes antigen presentation and tumor cell killing by macrophages, and may regulate the expression of MHC class II antigen (29). IL-4 has synergistic effects with GM-CSF, IL-3 and LPS, and can also induce peripheral blood mononuclear cells to secrete G-CSF and M-CSF, and enhance neutrophil-mediated phagocytosis, cell killing activity, and antibody-dependent cellular cytotoxicity (30). IL-4 is also a mouse macrophage chemokine that promotes the production of the interleukin-1 receptor antagonist (31). In this study, we found that DCs transfected with IL-12 could increase IL-4 expression, which may also inhibit tumor cell growth. For an example, IL-4 overexpression was found to suppress tumor development through p21-mediated activation of Janus kinase-signal transducer of activators of transcription (STAT) pathways in melanoma models (32); however, elucidation of the exact mechanisms involved in our experiment will require further investigation.

Patient DCs can specifically eliminate tumor cells and do not injure the majority of healthy cells (33), confirming the efficacy of immunotherapy using DCs. IL-12 is a cytokine with immunoregulatory activity and functions as an important link in the process of immune regulation (8). In this study, we overexpressed IL-12 in DCs, leading to enhanced anticancer effects against melanoma. Our results provide experimental evidence for future clinical application of immunotherapy in different types of cancer.

This study had some limitations. First, although overexpression of IL-12 also lead to increased IL-4, we did not clarify the exact mechanisms involved. Second, if possible, in vitro experiments should be also conducted to verify the function of DCs overexpressing IL-12 in the treatment of cancer. Overall, the exact mechanisms underlying the antitumor effects of DCs overexpressing IL-12 warrant further investigation.

In conclusion, an intratumoral injection of DCs overexpressing IL-12 was found to exert enhanced antitumor effects in melanoma. Biotherapy using DCs overexpressing IL-12 is a potential treatment strategy for melanoma.

\section{Acknowledgements}

Not applicable.

\section{Funding}

The present study was supported by the Science and Technology Planning Project of Jiangxi Science and Technology Department (nos. 20121BBG70052 and 20151BBA13053).

\section{Availability of data and materials}

The datasets used during the present study are available from the corresponding author upon reasonable request.

\section{Authors' contributions}

WY, YL and HW conceived and designed the experiments; WY, YL, LZ, XZ, ZZ and MZ performed the experiments and analyzed the data; WY, YL and HW wrote the manuscript. All authors read and approved the manuscript and agree to be accountable for all aspects of the research in ensuring that the accuracy or integrity of any part of the work are appropriately investigated and resolved.

\section{Ethics approval and consent to participate}

The experimental protocols were approved by the Ethics Committee of Jiangxi Provincial People's Hospital.

\section{Patient consent for publication}

Not applicable. 


\section{Competing interests}

The authors declare that they have no competing interests.

\section{References}

1. Thomson CH, Cassell O, Peach H, Holloway S, Garioch J and Moncrieff M: Neuropathic pain and quality of life after wide local excision and sentinel lymph node biopsy for melanoma: A multicentre study. Melanoma Res 27: 121-125, 2017.

2. de Vries IJ, Lesterhuis WJ, Barentsz JO, Verdijk P, van Krieken JH, Boerman OC, Oyen WJ, Bonenkamp JJ, Boezeman JB, Adema GJ, et al: Magnetic resonance tracking of dendritic cells in melanoma patients for monitoring of cellular therapy. Nat Biotechnol 23: 1407-1413, 2005.

3. Cheng Y, Ma XL, Wei YQ and Wei XW: Potential roles and targeted therapy of the CXCLs/CXCR2 axis in cancer and inflammatory diseases. Biochim Biophys Acta Rev Cancer 1871: 289-312, 2019.

4. Barry RJ, Tallouzi MO, Bucknall N, Mathers JM, Murray PI, Calvert MJ, Moore DJ and Denniston AK: Anti-tumour necrosis factor biological therapies for the treatment of uveitic macular oedema (UMO) for non-infectious uveitis. Cochrane Database Syst Rev 12: CD012577, 2018.

5. Mandalà M and Rutkowski P: Rational combination of cancer immunotherapy in melanoma. Virchows Arch 474: 433-447, 2019.

6. Summerfield A and McCullough KC: Dendritic cells in innate and adaptive immune responses against influenza virus. Viruses 1: 1022-1034, 2009.

7. Mellman I: Dendritic cells: Master regulators of the immune response. Cancer Immunol Res 1: 145-149, 2013.

8. Liu J, Cao S, Kim S, Chung EY, Homma Y, Guan X, Jimenez V and Ma X: Interleukin-12: An update on its immunological activities, signaling and regulation of gene expression. Curr Immunol Rev 1: 119-137, 2005.

9. Lasek W, Zagożdżon R and Jakobisiak M: Interleukin-12: Still a promising candidate for tumor immunotherapy? Cancer Immunol Immunother 63: 419-435, 2014.

10. Tagawa T, Albanese M, Bouvet M, Moosmann A, Mautner J, Heissmeyer V, Zielinski C, Lutter D, Hoser J, Hastreiter M, et al: Epstein-Barr viral miRNAs inhibit antiviral $\mathrm{CD}^{+} \mathrm{T}$ cell responses targeting IL-12 and peptide processing. J Exp Med 213: 2065-2080, 2016.

11. Cemazar M, Ambrozic Avgustin J, Pavlin D, Sersa G, Poli A, Krhac Levacic A, Tesic N, Lampreht Tratar U, Rak M and Tozon N: Efficacy and safety of electrochemotherapy combined with peritumoral IL-12 gene electrotransfer of canine mast cell tumours. Vet Comp Oncol 15: 641-654, 2017.

12. Komai-Koma M, Wang E, Kurowska-Stolarska M, Li D, McSharry $\mathrm{C}$ and $\mathrm{Xu} \mathrm{D}$ : Interleukin-33 promoting Th1 lymphocyte differentiation dependents on IL-12. Immunobiol 221: $412-417,2016$

13. Lu X: Impact of IL-12 in cancer. Curr Cancer Drug Target 17: 682-697, 2017

14. Li L, Jiang Y, Lao S, Yang B, Yu S, Zhang Y and Wu C: Mycobacterium tuberculosis-Specific IL-21 $1^{+} \mathrm{IFN}-\gamma^{+} \mathrm{CD} 4^{+} \mathrm{T}$ cells are regulated by IL-12. PLoS One 11: e0147356, 2016.

15. Feng C, Feng M, Jiao R, Liu D, Jin Y, Zhao X and Xiao R: Effect of Dezocine on IL-12 and IL-10 secretion and lymphocyte activation by culturing dendritic cells from human umbilical cord blood. Europ J Pharmacol 796: 110-114, 2017.

16. Thada S, Ponnana M, Sivangala R, Joshi L, Alasandagutti M, Ansari MS, Schumann RR, Valluri V and Gaddam S: Polymorphisms of IFN- $\gamma(+874 \mathrm{~A} / \mathrm{T})$ and IL-12 $(+1188 \mathrm{~A} / \mathrm{C})$ in tuberculosis patients and their household contacts in Hyderabad, India. Hum Immunol 77: 559-565, 2016.

17. Naito S, von Eschenbach AC, Giavazzi R and Fidler IJ: Growth and metastasis of tumor cells isolated from a human renal cell carcinoma implanted into different organs of nude mice. Cancer Res 46: 4109-4115, 1986.
18. Livak KJ and Schmittgen TD: Analysis of relative gene expression data using real-time quantitative PCR and the 2(-Delta Delta C(T)) method. Methods 25: 402-408, 2001.

19. Ribas A, Hamid O, Daud A, Hodi FS, Wolchok JD, Kefford R, Joshua AM, Patnaik A, Hwu WJ, Weber JS, et al: Association of pembrolizumab with tumor response and survival among patients with advanced melanoma. JAMA 315: 1600-1609, 2016.

20. See P, Dutertre CA, Chen J, Günther P, McGovern N, Irac SE, Gunawan M, Beyer M, Händler K, Duan K, et al: Mapping the human DC lineage through the integration of high-dimensional techniques. Science 356: eaag3009, 2017.

21. Bhattacharya P, Thiruppathi M, Elshabrawy HA, Alharshawi K, Kumar P and Prabhakar BS: GM-CSF: An immune modulatory cytokine that can suppress autoimmunity. Cytokine 75: 261-271, 2015.

22. Deng J,Li Y,Pennati A, Yuan S, Wu JH, Waller EK and Galipeau J: GM-CSF and IL-4 fusion cytokine induces B cell-dependent hematopoietic regeneration. Mol Ther 25: 416-426, 2017.

23. Lutz MB, Suri RM, Niimi M, Ogilvie AL, Kukutsch NA, Rössner S, Schuler G and Austyn JM: Immature dendritic cells generated with low doses of GM-CSF in the absence of IL-4 are maturation resistant and prolong allograft survival in vivo. Eur J Immunol 30: 1813-1822, 2000

24. Fukao T, Tanabe M, Terauchi Y, Ota T, Matsuda S, Asano T, Kadowaki T, Takeuchi T and Koyasu S: PI3K-mediated negative feedback regulation of IL-12 production in DCs. Nat Immunol 3: 875-881, 2002.

25. Kamensek U, Cemazar M, Lampreht Tratar U, Ursic K and Sersa G: Antitumor in situ vaccination effect of TNFa and IL-12 plasmid DNA electrotransfer in a murine melanoma model. Cancer Immunol Immunother 67: 785-795, 2018.

26. Oliveira Brito PK, Goncalves TE, Fernandes FF, Miguel CB, Rodrigues WF, Lazo Chica JE, Roque-Barreira MC and da Silva TA: Systemic effects in naïve mice injected with immunomodulatory lectin ArtinM. PLoS One 12: e0187151, 2017.

27. Sonabend AM, Velicu S, Ulasov IV, Han Y, Tyler B, Brem H, Matar MM, Fewell JG, Anwer K and Lesniak MS: A safety and efficacy study of local delivery of interleukin-12 transgene by PPC polymer in a model of experimental glioma. Anticancer Drugs 19: 133-142, 2008.

28. Kobiyama K, Temizoz B, Kanuma T, Ozasa K, Momota M, Yamamoto T, Aoshi T, Kuroda E and Ishii KJ: Species-dependent role of type I IFNs and IL-12 in the CTL response induced by humanized $\mathrm{CpG}$ complexed with $\beta$-glucan. Eur J Immunol 46: 1142-1151, 2016.

29. Yoshimoto T, Yasuda K, Tanaka H,Nakahira M,Imai Y,Fujimori Y and Nakanishi K: Basophils contribute to T(H)2-IgE responses in vivo via IL-4 production and presentation of peptide-MHC class II complexes to CD4 ${ }^{+}$T cells. Nat Immunol 10: 706-712, 2009.

30. Yeap WH, Wong KL, Shimasaki N, Teo EC, Quek JK, Yong HX, Diong CP, Bertoletti A, Linn YC and Wong SC: CD16 is indispensable for antibody-dependent cellular cytotoxicity by human monocytes. Sci Rep 6: 34310, 2016.

31. Helmy A, Guilfoyle MR, Carpenter KLH, Pickard JD, Menon DK and Hutchinson PJ: Recombinant human interleukin-1 receptor antagonist promotes M1 microglia biased cytokines and chemokines following human traumatic brain injury. J Cereb Blood Flow Metab 36: 1434-1448, 2016.

32. Lee HL, Park MH, Song JK, Jung YY, Kim Y, Kim KB, Hwang DY, Yoon do Y, Song MJ, Han SB and Hong JT: Tumor growth suppressive effect of IL-4 through p21-mediated activation of STAT6 in IL-4R $\alpha$ overexpressed melanoma models. Oncotarget 7: 23425-23438, 2016.

33. Sznol M: Betting on immunotherapy for melanoma. Curr Oncol Rep 11: 397-404, 2009. 